\title{
Une relecture des phénomènes transpositifs à la lumière de la didactique comparée
}

\section{Maria Luisa Schubauer-Leoni et Francia Leutenegger}

Les phénomènes de transposition didactique sont centraux dans l'étude des processus d'enseignement et d'apprentissage. Cette théorie, initiée par Y. Chevallard dans les années 1980, a été interrogée par les autres didactiques qui ont cherché à l'importer dans leur domaine. Depuis la didactique des mathématiques elle s'est développée vers une approche anthropologique des pratiques didactiques en poursuivant son programme épistémologique. Cet article, né d'un questionnement issu de la didactique comparée, cherche à mettre en lumière certains des aspects fondamentaux de la théorie ainsi que des points aveugles. La question du rôle attribué aux phénomènes de "personnalisation» et de "contextualisation" est tout spécialement discutée dans la perspective d'étudier ce qui se passe, ordinairement, dans les classes du point de vue didactique.

\section{L'action collective dans les œuvres et dans leur "reconstruction" scolaire}

La didactique comparée à laquelle nous souhaitons contribuer se situe dans le prolongement des didactiques disciplinaires. Sans elles, le projet comparatiste en didactique perdrait toute signification. Il s'agit dès lors d'avancer dans une approche théorico-méthodologique pour étudier les phénomènes d'enseignement/apprentissage d'objets spécifiques et construire, ce faisant, un domaine scientifique propre au sein des sciences didactiques et des sciences de l'éducation. En convoquant les sciences qui s'occupent des objets culturels, des sujets et des institutions, c'est un dialogue renouvelé au sein des sciences humaines et sociales qui est recherché.

Nous avons explicité ailleurs (Mercier, Schubauer-Leoni \& Sensevy, 2002; Schubauer-Leoni \& Leutenegger, 2002) les divers découpages qui caractérisent, à ce jour et selon nous, l'entrée dans ce champ d'étude. Par ailleurs, un certain nombre de travaux commencent à exister, dans le champ francophone, qui se réclament de ce type de problématisation comparatiste (Mercier et al. , 2002). Dans le cadre de la présente contribution, nous allons privilégier la discussion 
d'un concept majeur de la didactique des disciplines: la transposition didactique. Concept générique par excellence, il nécessite la prise en compte de ses déclinaisons selon les objets d'enseignement/apprentissage spécifiques. A ce titre, il désigne les conditions de possibilité du travail/étude dans la classe de questions culturelles précises inhérentes à différents savoirs.

Si l'appellation de transposition didactique a largement été employée dans les travaux didactiques, le sort réservé à la théorie de la transposition et à son remaniement depuis les années 1980, nous a paru significatif de l'évolution des problématiques didactiques. Nous ne pourrons pas développer ici ce que devient le concept tel que réinscrit dans le débat interne à chaque discipline afin de dégager, démarche documentaire à l'appui, les dimensions communes et distinctives à ces collocations. Un travail approfondi reste à faire dans cette optique bien que quelques analyses aient, d'ores et déjà, posé des jalons importants dans ce sens (Bronckart \& Plazaola Giger, 1998; Johsua, 1996 et 1998; Mercier, 2002; Raisky \& Caillot, 1996).

Nous nous centrerons sur deux aspects: premièrement les débats issus des différentes didactiques au sujet du concept, au regard des objectifs qu'elles se donnent -unanimement-lorsqu'elles prétendent traiter de l'articulation entre enseignement et apprentissage de savoirs scolaires ; deuxièmement le rôle et le poids relatif attribué, par les approches en termes de transposition didactique, aux sujets (versus aux personnes) et aux institutions dans le processus d'enseignement/apprentissage. Il s'agit pour nous d'interroger d'une autre façon les épistémologies sous-jacentes aux phénomènes transpositifs tels que traités par les didacticiens.

Plus précisément, cette contribution cherche à montrer l'intérêt qu'il peut y avoir à situer le lieu d'investigation des phénomènes de transposition didactique ni (que) dans les savoirs ni (que) dans les sujets -enseignants et apprenants- mais dans leur action conjointe. Il s'agit ensuite de considérer ce travail de coproduction de connaissances à la lumière des pratiques culturelles qui le légitiment. Cette entrée pose dès lors l'action collective et ses effets reconnus au centre de la problématique transpositive. Le processus de transposition est ainsi pensé comme constitutif à la fois de la création renouvelée d'ouvres et, dans le même mouvement, des personnes qui y contribuent. En d'autres termes, nous estimons que la genèse sociale et historique des ouvres et la genèse scolaire et artificielle des savoirs transposés doivent être traitées en assurant la cohérence épistémologique des critères de description et de compréhension des phénomènes en jeu.

Avant d'entrer dans la discussion de ce point central, considérons brièvement l'état du questionnement dans différentes didactiques en matière de transposition. Ceci devrait nous permettre de prendre la mesure de l'importance du travail à impulser dans une perspective comparatiste. 


\title{
Un concept nomade: Les débats issus des différentes didactiques
}

\author{
- "C'est quoi déjà la transposition didactique? \\ - Mais tu te rappelles, c'est cette histoire de distance entre le \\ savoir savant et le savoir enseigné..."
}

Cet échange pourrait être celui de deux étudiants débutants dans le domaine didactique... Or, cette histoire de distance a interpellé nombre de didacticiens. Divers chercheurs ont signifié leur intérêt pour un concept qui leur semblait venir à propos pour avancer dans leurs propres problématisations didactiques. Leurs travaux attestent ainsi de l'intégration du concept dans les systèmes théoriques auxquels ces chercheurs contribuent. Citons notamment:

- du côté de l'éducation physique: Amade-Escot (2003);

- pour la didactique de l'histoire et de la géographie: Audigier (1988) ; Audigier, Crémieux et Tutiaux-Guillon (1994);

- pour les sciences économiques et sociales: Beitone et Legardez (1995); Beitone, Decugis, Dollo et Rodrigues (2004); Dollo (2005);

- pour la didactique du français: Bronckart et Plazaola-Giger (1998); Bronckart et Schneuwly (1991); Canelas Trevisi (1997); Schneuwly (1995);

- du côté de la didactique de la physique: Johsua (1985); Tiberghien (1989);

- en didactique professionnelle: Rogalski et Samurcay(1994);

- sans oublier les nombreux travaux conduits en didactique des mathématiques dans le sillage de l'œuvre princeps (pour une revue d'ensemble des études de ce champ et de leurs conditions d'émergence voir Mercier, 2002).

Après vingt ans de vie et de nomadisme de la notion dans les communautés didacticiennes qui ont grandi et parfois émergé avec elle, force est toutefois d'admettre que les ingrédients de la théorie initiale ont souvent été naturalisés, rigidifiés avec pour effet une cristallisation autour de (ou contre les) quelques éléments primitifs. Rares sont les contributions qui interrogent des composantes épistémologiques du modèle et carrément absents les travaux qui questionnent, depuis d'autres didactiques disciplinaires, l'évolution de la problématique de la transposition didactique en didactique des mathématiques. D'une façon générale, on assiste plutôt à un travail de vérification de la compatibilité de la première théorie chevallardienne avec une certaine lecture, personnelle, des réalités d'un domaine d'enseignement, ce qui produit une floraison de schémas plus ou moins alternatifs qu'on peut interpréter comme autant de moyens pour s'approprier la théorie en l'adaptant au cadre du commentateur. Souvent, même si le ton est âpre, les critiques restent sans objet fondamental et la question transpositive persiste.

\section{Les enjeux d'une filiation}

Le plus souvent, les didacticiens qui se réferent à la transposition didactique rattachent l'étude didactique inaugurale de Chevallard (1985/1991) à celle, socio- 
logique, de Verret (1975). Nous avons fait de même (Schubauer-Leoni, 1998), la filiation semblant aller de soi pour ceux qui découvrent Verret dans l'ouvrage de Chevallard $^{1}$. A notre décharge disons que l'ouvrage est peu disert sur le projet scientifique sociologique de Verret et sur le déplacement opéré, depuis cette problématique, vers une théorisation en didactique des mathématiques. Il s'agit, désormais, de tenir compte aussi de l'évolution de la théorie et des éclairages que les textes plus récents de Chevallard apportent à l'œuvre initiale. Nous convenons dès lors, avec d'autres commentateurs (Terrisse \& Léziart, 1997; Mercier, 2002; Astolfi, 2005), que Verret forge le concept de transposition didactique en l'associant à la nature bureaucratique de l'enseignement, dans un procès aux mécanismes de transmission des savoirs philosophiques chez les étudiants de Sciences Humaines à l'université (étudiants qui, par leurs origines sociologiques, ne se trouvent pas tous dans le même rapport à la règle scolaire et à l'urgence temporelle qu'elle comporte). C'est ce type d'enseignement qui s'avère marqué par les fameuses contraintes appelées: désyncrétisation; dépersonnalisation; programmabilité de l'acquisition; publicité du savoir; contrôle social des apprentissages. Dans la problématique de Verret de telles contraintes engendrent l'évitement de la rencontre des étudiants (principalement ceux des couches défavorisées) avec les œuvres philosophiques elles-mêmes. Ce type d'organisation de l'enseignement est alors désigné par contraste avec une organisation «aristocratique» de l'enseignement qui ne serait permise qu'aux étudiants issus des couches les plus favorisées. Comme nous le rappelle Mercier (2002), Verret est prêt à penser que certaines disciplines -celles qu'il estime pouvoir supporter la programmation des savoirs: les mathématiques notamment- s'accommodent plus que d'autres de l'école bureaucratique! Or, toujours en suivant Mercier, la «rupture fondatrice» introduite par Chevallard en 85 consiste à dire que le coût des systèmes d'enseignement modernes depuis le 19ème siècle nécessite que toutes les matières subissent une forme d' "apprêt didactique», les mathématiques n'étant pas plus "programmables» que d'autres disciplines. C'est cette position qu'exprime Chevallard dans la post-face de l'ouvrage réédité en 1991. Nous allons ainsi volontiers dans le sens de Astolfi (2005) qui estime que «Verret plaide alors pour un retour à la vérité de la science» en inscrivant dès lors le concept dans une théorisation de la reproduction sociale sans d'ailleurs que son concepteur lui attribue une fonction centrale dans la suite de ses propres travaux ${ }^{2}$.

Or, nous l'avons précisé, l'entreprise comporte un autre enjeu pour le champ didactique: la transposition didactique, dès son effet d'annonce, tend à fonder une théorisation didactique capable de supporter l'entrée nécessaire du " savoir " dans les questions d'enseignement et d'apprentissage. En tant que concept central, il constitue l'ossature première de l'édifice didactique chevallardien qui s'ouvrira à une approche dite anthropologique. 


\section{Les débats relatifs à la théorisation initiale de la transposition didactique}

Revenons brièvement sur quelques points théoriques qui ont cristallisé les critiques et sur lesquels nous souhaitons ensuite appuyer notre thèse: pour nous, c'est une entrée du côté de l'action conjointe 3 qui permet le mieux, de penser le processus transpositionnel en traitant en même temps des objets et des sujets au sein des institutions. Par ce biais nous interrogerons les développements ultérieurs de la théorie de la transposition didactique tels que traités par Chevallard et collaborateurs ${ }^{4}$ tout en désignant des points qui semblaient aveugles dans l'approche de 85. Nous commencerons donc par les prises de position en provenance d'autres didactiques qui s'en sont tenues, pour l'essentiel, au modèle initial.

Posé en rupture avec les approches psychopédagogiques, le projet didactique à l'enseigne de la transposition a affiché d'entrée de jeu un modèle descendant. Le mouvement qui semble aller de soi est celui qu' esquisse le sous-titre de l'ouvrage: «Du savoir savant au savoir enseigné». L'auteur s'expliquera à ce propos dans la post-face de l'édition de 91, mais d'emblée cette problématisation de haut en bas a engendré de vastes polémiques dont Mercier (2002), Johsua (1996), Bronckart et Plazaola Giger (1998) et d'autres encore, ont déjà rendu compte. Un certain agacement exprimé par plusieurs didacticiens provient vraisemblablement de l'idée que l'on puisse généraliser un modèle qui n'aurait de sens, à leurs yeux, que pour les mathématiques. Par ailleurs, la posture althusserienne et la référence systématique à Bourdieu et -à l'arrière plan- à la sociologie de Durkheim, y donnent un aperçu d'une théorie où le maître-mot est celui de contrainte. Nous considérons volontiers que le trait est apparu d'autant plus forcé qu'il s'agissait pour Chevallard de rompre avec une vision des réalités scolaires qui incitait au volontarisme innovant.

Dans l'esprit de la théorie de la transposition, le concept d'ingénierie didactique a été un moyen de répondre à des questions émanant du terrain de l'enseignement sans adopter le langage de l'innovation. L'ingénierie est, dans ce cas, au service à la fois de la recherche fondamentale de la science didactique et des demandes en provenance des pratiques enseignantes. La production de phénomènes didactiques, rendus observables par les conditions de l'ingénierie, sert le développement scientifique de la didactique et les projets de modification des pratiques sont, dans ce cas, pensés comme devant être menés sous le contrôle épistémologique du travail d'ingénierie.

Prônant une posture objectivante à l'égard du monde scolaire à travers la célèbre formule de Durkheim selon laquelle il convient de prendre les faits sociaux comme des " choses " dotées a priori de caractéristiques propres, c'est l'image d'une théorie déterministe, pour ne pas dire positiviste, qui prend du relief. Une lecture de ce type rend difficile la perception, dans l'esquisse initiale de la transposition, des composantes transactionnelles porteuses de libertés du côté des sujets 5 . La "constitution» (au sens althussérien) des individus concrets en sujets du système didactique nourrit vraisemblablement quelques soupçons de la part de 
certains lecteurs de la théorie de la transposition didactique. En effet le choc est grand, en tant qu'intervenants dans les systèmes d'enseignement, d'être confrontés, en même temps, à la question de la légitimité des objets enseignés et appris et à la question de l'assujettissement des personnes en position d'enseignant et d'enseignés.

Voyons les enjeux de plus près:

- Du côté de la légitimité des objets, il s'agit désormais d'en travailler l'origine extrascolaire pour en assurer la justification au sein des systèmes d'enseignement et des divers systèmes didactiques. C’est la «valeur» même des savoirs enseignés qui est en jeu. Le propos de Halté (1998) est significatif: «[...] en posant que le savoir réellement enseigné n'est pas le savoir savant de départ, pire, ne peut pas l'être, elle (la transposition didactique) donne le désagréable sentiment d'une déformation, d'une dégradation inéluctable...» (p. 173). D'autres auteurs prendront des positions assez proches. Parmi les positions les plus radicales, nous trouvons celle de Tozzi (1995) qui met en avant la nature de l'objet «philosophie» pour contrer la pertinence même du concept de transposition didactique: «[...] le sens de la vie et de la mort, l'amour, le devoir ou le bonheur par exemple ont le même degré de proximité essentielle pour chacun, que l'on s'appelle Socrate ou Dupont» (p. 241). Une autre position, en matière d'enseignement de la philosophie est notamment celle défendue par Bautier \& Rochex (2004) qui soutiennent, en s'appuyant sur la théorie des genres de discours (genres premiers, genres seconds) de Bahktine, la thèse d'une secondarisation nécessaire des objets et des pratiques; ces genres secondaires étant au principe même des situations et des activités scolaires (Schneuwly, 1994).

- Du côté de l'assujettissement des personnes, enseignant et enseignés, il s'agit de prendre la mesure des contraintes des systèmes diffuseurs de savoirs organisés en disciplines scolaires. Dans les débats issus des diverses didactiques, la notion de «savoir savant» 6 est discutée d'une part pour dire que si elle est peut-être pertinente en mathématiques, elle ne l'est pas dans d'autres disciplines qui ne disposent pas d'une seule et unique référence stabilisée de type savant (Halté, 1992, pour la didactique du français; Chatel, 1995, du côté des sciences économiques et sociales; Audigier, Crémieux et Tutiaux-Guillon, 1994, pour la didactique de l'histoire et de la géographie); d'autre part pour rappeler que d'autres pratiques sociales existent qui ne sont pas assimilables à des «savoirs savants» tout en jouant un rôle de référence pour les enseignements à l'école ${ }^{7}$. Cette dichotomie et le débat qu'elle engage risquent ainsi de véhiculer l'idée selon laquelle d'un côté les savoirs dits «savants» ne relèveraient pas de pratiques sociales (ce qui tend à fournir une vision substantialiste des œuvres culturelles ${ }^{8}$ ) et d'un autre côté que les "pratiques sociales» seraient réduites à des expériences pratico-pratiques dont l'accès serait permis uniquement par une sorte de fréquentation directe, voire personnalisée des praticiens. Quelques voix (Johsua, 1996, depuis la didactique des mathématiques et des sciences; Schneuwly, 1995, en tant que didacticien du français) s'élèvent toutefois pour rappeler que, dans tous les cas, la communication 
didactique entre enseignant et élève ne peut se faire que par le biais d'une modélisation des pratiques ce qui revient à forger un savoir sur les pratiques.

A noter que ces prises de position ont notamment eu le mérite d'amener des distinctions entre différents types de savoirs et partant entre institutions chargées de créer les conditions d'accès à ces savoirs. Samuel Johsua (1998), afin de discuter "des savoirs et de leur étude» distingue les «savoirs pratiques» des «savoirs techniques». On apprendrait les premiers de façon «silencieuse» en dehors de toute intentionnalité hétéro-didactique au sein d'une institution scolaire. Il cite à ce propos l'exemple de l'apprentissage de la marche voire de telle «démarche» particulière et culturellement marquée. L'accès "par immersion» serait caractéristique de l'approche "en action" de tels savoirs pratiques. Les seconds devraient faire l'objet d'une étude systématique et explicitement pensée comme telle par la personne qui s'y engage. L'exemple de l'apprentissage du vélo sert à l'auteur à montrer la présence d'une intentionnalité produisant les essais et erreurs typiques de cet apprentissage qui ne nécessite pas (sauf à vouloir en faire une activité professionnelle) une institution organisatrice de l'accès à l'étude de cette pratique. Parmi les savoirs techniques, Johsua distingue encore ceux «hautement techniques» dont la rencontre ne peut être faite sans la mise en place d'une organisation apte à produire une didactification de ces savoirs. C'est typiquement le cas des mathématiques où l'accès par immersion dans le monde de questions utiles à la construction de l'édifice paraît fort improbable en dehors d'un directeur d'étude qui organiserait, sciemment, les classes de questions utiles aux différentes étapes de l'apprentissage. L'aide à l'étude qu'organisent expressément les institutions didactiques (pour des savoirs techniques et hautement techniques) suppose donc un " apprêt didactique " des objets d'enseignement. Comme le précise Johsua, une telle organisation pour l'aide à l'étude est typiquement un aspect du processus de transposition didactique. Le cas de la formation professionnelle, où l'institution de référence essentielle est constituée par les experts de la profession mériterait un détour en soi dans l'ensemble des distinctions opérées. Les travaux de didactique professionnelle (Rogalski, 2004; Rogalski \& Samurçay, 1994; Veillard, 2000) constituent à ce titre une excellente occasion de décentration par rapport aux entrées «scolaires» habituelles en matière de transposition didactique.

\section{Le "texte du savoir» et les phénomènes de contextualisa- tion et personnalisation des savoirs}

Au-delà du cas des mathématiques et selon l'origine (" savante » ou " experte ») des savoirs à enseigner, le processus de transposition didactique et ses phénomènes de décontextualisation-recontextualisation, ainsi que de dépersonnalisationrepersonnalisation sont considérés incontournables ${ }^{9}$. Nous y reviendrons. Mais pour cela nous devons considérer d'abord une autre composante, encore partiellement verrouillée dans les débats inter-didactiques et fortement liée aux phénomènes de décontextualisation et de dépersonnalisation: la question du «texte du 
savoir». Par cette expression le Chevallard de 1985 évoque un aspect central du processus d' «apprêt didactique». Ce processus paraît se figer sous la forme de textes écrits (manuel, instruction officielle, ...) et concrétise, par ce biais, la «mise en éléments» des composantes d'un corps de savoir qui se présente (dans les pratiques de référence) comme syncrétique. Cette nécessité de textualisation de la transposition didactique et son lien avec le processus de dépersonnalisation a notamment amené Bronckart et Plazaola-Giger (1998) à contester la «position subjectiviste» que ce lien véhicule. Ces commentateurs disent notamment que la notion de dépersonnalisation laisse croire que «les discours et les savoirs seraient liés à une personne singulière» ce qui leur paraît «incompatible avec l'orientation épistémologique qui sous-tend l'ensemble de l'ouvrage». Plus profondément, ces auteurs rediscutent le rapport textes-savoirs en soulignant à quel point l'accès aux savoirs est sémiotisé et véhiculé dans les textes. Mais alors il devient nécessaire, rappellent-ils, de spécifier les genres de textes selon les formes communicatives spécifiques et les formations sociales qui engendrent des déclinaisons en types de discours. Si l'on admet avec Bronckart (1997) que les modalités de prise en charge énonciatives sont constitutives de certaines formes de matérialisation de l'activité de pensée, nous estimons que l'étude de la transposition didactique ne peut éviter la prise en compte de telles modalités dans les processus générateurs de connaissances privées ${ }^{10}$ et de savoirs publics. La notion de personnalisation empruntée à Verret nourrit l'image d'une culture faite d'œuvres monumentales de tel Auteur ou alors l'opération de dépersonnalisation est telle que le savoir est détourné de toute pratique humaine et sociale et se présente désincarné sous la forme d'une pure pensée. Dans les deux cas, le risque est grand de produire un rapport de révérence à une œuvre perçue comme fermée et donc non questionnable.

Ce point nous paraît fondamental pour le développement de la théorie de la transposition en didactique: sans poser la question de la genèse socio-historique et institutionnelle des œuvres, et donc des pratiques sociales et culturelles qu'elles comportent, on ne peut ni penser une genèse "artificiell " à travers laquelle des élèves pourraient redécouvrir des éléments essentiels des œuvres de référence ${ }^{11}$, ni comprendre ce qui se passe et se joue dans le didactique ordinaire. Dans une classe "quelconque», il s'agit en effet de comprendre, à partir des systèmes de tâches proposés aux élèves, dans quelles pratiques scolaires effectives ils sont engagés, avec quels effets sur le plan des connaissances et avec quel lien de légitimité à l'égard des pratiques de référence.

Dans tous les cas, à des fins d'enseignement, le savoir à enseigner est mis en scène, les objets qui le composent, sont parlés, schématisés, notés au tableau et dans des cahiers, qui recréent un univers de référence pour/par l'action d'enseignement (traces à l'usage du professeur) et pour/par l'action d'apprentissage (traces pour l'étude de l'élève). Dans la théorie de la transposition, la fonction de ces «mises en textes» est essentielle et constitutive du processus de réorganisation des objets d'enseignement et d'apprentissage et de l'action conjointe à leur propos. 
De fait, l'analyse de la transposition didactique met en évidence des «euilletés» de textes: certains préexistent à l'action conjointe et la préfigurent partiellement dans la mesure où ces textes dans leur version écrite (les documents à l'usage des profs, les guides, ...) et dans leur version orale (les théories de la culture professionnelle, les projets qu'elle engendre) disent l'action à entreprendre ; d'autres textes émergent de l'action conjointe du professeur et des élèves. Dans tous les cas, ces textes, dans le processus-même de leur interprétation/élaboration, sont porteurs de temporalité et en ce sens produisent des savoirs-temps.

Bien que l'ouvrage de 1985, réédité en 1991, soit très peu explicite sur le travail effectif d'étude des phénomènes de transposition, la désignation de lieux de mise en texte (traces écrites principalement) a suffi pour que des chercheurs, issus de diverses didactiques, engagent des travaux sur la distance (ou le décalage) entre textes officiels disant le découpage des programmes et manuels ou/et entre savoirs de référence et savoirs à enseigner dans les manuels (transposition didactique externe) (voir Marschall, Plazaola Giger, Rosat \& Bronckart, 2000, pour un exemple dans le domaine de l'enseignement des langues 2). Nettement moins nombreux sont en revanche les études qui ont exploré ce qui se passe effectivement dans les classes (transposition didactique interne) en regard de ce qui est prescrit en amont (Canelas Trevisi, Moro, Schneuwly \& Thévenaz, 1999; Mercier, 1997; Leutenegger, 2003). Le texte co-élaboré dans l'échange didactique s'avère précipité dans toutes sortes d'enjeux interpersonnels et de groupes en fonction de normes, valeurs, conventions qui dépassent le projet strict de traitement de telle question didactique. Le contrat didactique s'immisce dans la gestion du contrat de communication au sens de Charaudeau (2004). Le didactique diffuse dans le pédagogique et l'éducatif.

\section{Le rôle et le poids relatif attribués aux sujets et aux institutions dans le processus d'enseignement/apprent issage}

\section{L'approche anthropologique}

La première étape de la théorie de Chevallard annonçait un caractère résolument épistémologique: la description du régime didactique du savoir. Lapproche, qui se défend de toute prescription, permet de voir que cette posture, pourtant nécessaire, est difficilement tenable dans le monde éducatif où la demande sociale appelle un positionnement " pour » ou " contre " les phénomènes mis à jour par la recherche. Le paradoxe est d'autant plus fort que l'analyse dévoile ce qu'elle déclare comme devant rester caché: «l'illusion de l'identité de l'objet de savoir et de l'objet d'enseignement est nécessaire au fonctionnement heureux des systèmes didactiques» (Mercier, 2002). Mais le dévoilement ne concerne pas que le savoir. Confrontés à la description des phénomènes de transposition, l'enseignant aussi bien que les autres partenaires de la scène éducative (les formateurs, les inspec- 
teurs) ne peuvent que se sentir fragilisés dans leur rapport à des contenus d'enseignement qu'ils croyaient maîtriser. La culture de la description, sans prescription, des processus d'enseignement et d'apprentissage étant peu partagée dans le milieu éducatif, l'analyse des contraintes qui pèsent sur la dynamique des systèmes didactiques alliée à l'importance attribuée aux enjeux spécifiques à une discipline donnée peut laisser entendre que les réalités telles qu'elles sont décrites correspondent à celles souhaitées par les chercheurs didacticiens. De là à dire que le didacticien est conservateur, voire contre toute innovation, il n'y a qu'un pas!

Le développement de la théorie chevallardienne par la prise en compte des $d i$ mensions écologiques d'abord, puis par ce que l'auteur nommera la théorie anthropologique du didactique (TAD) ne modifient pas fondamentalement la position des réfractaires à la version "assez sommaire» ${ }^{12}$ de la théorie de la transposition didactique de départ. Ces développements qui, non seulement veulent élargir la théorie initiale mais visent à fournir des instruments opératoires pour l'étude des processus transpositifs, ne diffusent pas vraiment en dehors de la didactique des mathématiques. Il faut dire, qu'entre temps, les didacticiens des mathématiques et des autres disciplines se sont donnés leurs propres moyens d'investigation des textes et de diverses traces du processus de transposition.

Nous ne nous arrêterons pas spécifiquement sur les dimensions écologiques (Chevallard, 1989, 1992) sauf pour rappeler qu'elles initient l'espace de pensée anthropologique à travers la notion de rapport. Cette notion permet d'approcher les pratiques sociales qui mettent en jeu des objets sous couvert d'institutions. Les personnes (en position de sujets et donc à la fois assujettis aux institutions et producteurs de celles-ci) entretiennent des rapports à l'égard des objets en agissant sur et avec eux.

Dans le cadre de l'approche anthropologique, la notion d'activité (en l'occurrence mathématique) est déclarée centrale. Mais que désigne-t-on par activité mathématique? "Pour expliquer les faits d'enseignement auxquels elle se voit confrontée, la didactique postule que le «mystère» est dans les mathématiques, et non pas dans les sujets qui ont à apprendre et à enseigner les mathématiques» écrivent Bosch et Chevallard (1999, p. 79). La description des pratiques institutionnelles faisant appel à des objets mathématiques est alors traitée en termes d'organisations praxéologiques ${ }^{13}$. Plus précisément et en maintenant une posture résolument matérialiste, l'activité est décrite à l'aide des instruments matériels (papier, crayon, tableau, craie, règle, compas, ordinateur...) auxquels viennent s'ajouter d'autres objets tels que les écritures formalismes, graphismes, écritures, mots, discours, etc. Contre une vision idéaliste et jugée dominante des mathématiques dont l'essence serait dans les concepts, les auteurs mettent en avant l'importance «...de considérer la manière dont l'activité mathématique est conditionnée par les instruments matériels, visuels, sonores et tactiles qu'elle met en jeu» (p. 90). C'est alors sous couvert d'une dialectique entre les objets ostensifs et les objets non ostensifs qu'est caractérisée l'activité mathématique: les premiers renvoient aux objets qui assument une certaine matérialité perceptible (sons, gra- 
phismes, gestes...) et qui sont dès lors manipulables, les seconds expriment l'existence de notions, de concepts dont la manipulation est d'un autre ordre. L'activité humaine relève, dans cette acception, d'une "co-activation d'objets ostensifs et non ostensifs» (p. 92), la manipulation des premiers étant «réglée» par les seconds qui ne se donnent «à voir» ni à l'observateur ni aux acteurs eux-mêmes.

Selon Bosch et Chevallard (1999), le son est "émis et reçu», le graphisme est «tracé et lu», un geste est "fait et perçu». Pourtant, si ces expressions permettent de matérialiser et rendre perceptible, pour soi et pour autrui, le produit de l'action, en revanche cette description ne laisse aucune place au travail d'interprétation ni du sujet «émetteur» ni du «récepteur». L'activité mathématique appelle bien une description, mais celle-ci est inhérente aux possibles et aux contraintes de la culture mathématique telle qu'elle existe dans une institution donnée; elle est donc explicitement détachée du «fonctionnement cognitif des sujets qui la réalisent» (p. 116). La TAD est clairement une théorie épistémologique qui élabore un modèle de «ce que sont les connaissances mathématiques dont on a besoin et que l'on construit lorsqu'on fait des mathématiques» (Bosch \& Gascon, 2002, p.28).

L'inscription de la théorie de la transposition dans l'approche anthropologique du didactique se situe donc clairement dans le prolongement de ses prémices de 1985 et renouvelle ainsi le primat du programme épistémologique (au sens de Gascon, 1998) contre ce que les auteurs (Gascon, 1998; Bosch, Fonseca \& Gascon, 2004) appellent le programme cognitif en référence, notamment, aux travaux de Tall (1991) visant la création d'un modèle des processus cognitifs qui interviennent dans la construction de certains concepts mathématiques. Le programme épistémologique se positionne aussi par contraste avec l'approche cognitive de l'apprentissage des mathématiques chez Duval (1996) ${ }^{14}$. Nous comprenons les raisons qui amènent à se distancer d'une vision cognitiviste de l'activité qui éviterait la problématisation des connaissances mathématiques au profit du seul traitement interne du sujet apprenant. Nous estimons toutefois que le primat attribué à la prise en compte d'un «sujet assujetti», sorte de sujet générique ou épistémique potentiellement doté des techniques d'études utiles ou disponibles dans les institutions, ne peut longtemps être tenu si l'on veut articuler le programme épistémologique avec un programme didactique (au sens de Mercier, 2002) qui travaillerait sur les activités mathématiques telles qu'investies effectivement par des élèves concrets aux prises avec les tâches fournies par leurs professeurs. Tout en mettant en avant le programme épistémologique, l'approche anthropologique ne renonce toutefois pas à cette entrée du côté des activités effectives en classe (Bosch, Espinoza \& Gascon, 2003).

\section{Un programme didactique articulé à un programme épis témologique}

Dans le mouvement esquissé par les travaux sur le modèle de l'action du professeur $^{15}$, c'est la logique ascendante qui est privilégiée pour traiter des phénomènes 
transpositionnels. L'étude de l'action conjointe du professeur et des élèves renvoie à une épistémologie des possibles du côté du système didactique lorsque des sujets (enseignant et élèves) sont confrontés à des tâches mathématiques (problématisées au sens du programme épistémologique). Or, sans un modèle de construction des personnes susceptibles de s'assujettir, à des moments précis de leur vie, aux contraintes des institutions tout en travaillant leur propre espace de liberté et, partant, leur condition d'émancipation, le programme didactique perd en consistance et les fondements d'existence et de renouvellement des œuvres sont fragilisés. Cette position revient à dire que le processus de dépersonnalisation et de décontextualisation qui caractérise la dynamique transpositive nécessite que l'on donne un statut fort aux effets de repersonnalisation et de recontextualisation que le processus de transposition didactique engendre. En d'autres mots, ceci signifie que le processus de transposition didactique ne peut admettre un vide au plan de la personnalisation et de la contextualisation. L'enjeu théorique est donc, de notre point de vue, l'articulation entre le programme épistémologique et un programme didactique constitué par l'action conjointe du professeur et des élèves à propos des systèmes d'objets (mathématiques et non mathématiques) effectivement rencontrés au cours de leur agir dans une institution donnée. Ce programme ne peut dès lors être compatible avec le programme cognitif en tant que modélisation d'un prétendu fonctionnement interne d'un sujet isolé du système didactique et des attentes qui le caractérisent.

C'est la compatibilité entre programmes épistémologique et didactique qui doit dès lors être traitée. Bosch, Fonseca et Gascon (2004) déclarent que le programme épistémologique assume comme hypothèse une "dépersonnalisation de la problématique didactique», en plaçant au premier plan l'étude de «l'activité mathématique institutionnalisée», afin de chercher les causes mathématiques de certaines difficultés. Quant à nous, nous estimons que si le programme épistémologique est nécessaire, il ne peut que prendre appui sur un programme didactique qui travaille l'activité via l'agir d'individus concrets. Le programme épistémologique relève dans cette acception d'une forme d'analyse a priori qui convoque une activité mathématique possible et attendue dans une institution donnée (analyse descendante). Dans le programme didactique, cette analyse est suspendue momentanément au profit de l'agir effectif des sujets précipités dans les pratiques institutionnelles. Lanalyse (ascendante) de cet agir - en situation - devient ainsi un révélateur des caractéristiques spécifiques des conditions susceptibles de permettre la rencontre de tel savoir. Lactivité, dans le prolongement des travaux de Leontiev et Vygotski, et des analyses dont ils ont fait l'objet (Bronckart, 2000; Bronckart, Bulea, Filliettaz, Fristalon, Plazaola Giger \& Revaz, 2004; Friedrich, 1999), n'est pas dissociable, dans cette perspective, des phénomènes de conscience et d'interprétation de l'agir de la part des sujets/agents. Sous couvert d'institutions didactiques, l'enjeu concerne la nature des expériences provoquées didactiquement en référence aux expériences constitutives de l'œuvre. Ces occasions d'expériences émergent via les situations (organisatrices de questionnements) que l'élève rencontre, avec d'autres, en classe. 
Quel est alors le statut de l'expérience par rapport à l'action? Quelles sont les expériences (cruciales mais aussi ordinaires ${ }^{16}$ ) qui modifient les connaissances personnelles? En reprenant à notre compte la notion d' «agentivité» de Bruner (1991) pour signifier «une action dirigée vers un but, contrôlée par des agents» (p.88), nous considérons que, en fonction d'actions possibles, dans une situation reconnue comme prototypique et au sein d'une institution donnée (Sensevy, 2001) puissent émerger deux types d'expériences fortement articulées: l'expérience stricto sensu et l'expérience lato sensu.

L'expérience stricto sensu concerne l'agent 17 confronté à une tâche/situationproblème précise. Dans cette contingence, l'expérience prend forme si l'agent: accepte d'assumer ses propres responsabilités au sein du processus de dévolution concernant tel problème ; s'attribue des tâches en interprétant la situation ; se donne des buts, anticipe (orientation de l'action, réalisation de l'intention); procède par inférences (calcul d'actions possibles, recherches d'informations, contrôle de l'activité...); raisonne pratiquement (conceptualisation en acte à vocation pragmatique). Cette expérience comporte aussi des commentaires sur l'activité réalisée ou en cours de réalisation (dimension réflexive). L'expérience d'agentivité implique d'ailleurs aussi un processus d'auto/hétéro appréciation dont la teneur repose sur l'interprétation des attentes contractuelles. L'ensemble de cette praxis liée à l'effectuation de la tâche et aux discours qui l'accompagnent (verbalisations de l'action, formulations adressées à un co-agent, discours porteurs de -tentatives de- preuve, ...) comporte l'articulation avec une composante supplémentaire et non moins décisive pour l'expérience de l'agent: la tension entre public et privé. En effet, le discours de commentaire sur le travail en cours peut prendre la forme d'un langage intrapersonnel (on se parle à soi-même) sans donc que quiconque puisse y accéder; ou alors le discours prend la forme d'apartés avec le/la camarade installé/e à côté (co-agent). On peut considérer que cet échange interpersonnel prolonge une modalité «privée» de travail dans la mesure où il n'est pas accessible à l'enseignant. En revanche, d'autres formes discursives sont clairement de type public: pensons aux apartés entre l'enseignant - qui veut s'assurer du sens (et/ou de la conformité) de telle réponse- et l'élève, mais pensons surtout aux prestations d'un élève appelé au tableau pour écrire/dire sa réponse ou sa stratégie de résolution du problème devant la classe (Leutenegger, 2000 et 2003 ; Ligozat, 2003 ; Schubauer-Leoni, Leutenegger \& Mercier, 1999).

L'expérience lato sensu est constituée par la réorganisation des expériences stricto sensu telles que réalisées dans diverses institutions. Si l'agent vit l'expérience stricto sensu sous l'égide de l'assujettissement à une institution locale dans une conjoncture de travail précise, l'expérience lato sensu donne du souffle à l'agent, la personne émerge, l'expérience se fait histoire personnelle (et non pas seulement cumul d'histoires institutionnelles). La structure personnelle crée donc les conditions pour affronter de nouvelles postures d'agent lorsque vont se présenter les expériences stricto sensu subséquentes. Le processus d'émancipation, d'autonomisation de la personne prend forme et l'agent peut se muer en acteur 
qui joue avec et dans les interstices institutionnels. La viabilité et le renouvellement des institutions dépend de ce processus qui implique diverses catégories d'agents dans des postures spécifiques: certaines carrément instituées et voulues comme telles (cf. les topos distincts et complémentaires d'enseignant et d'enseigné); d'autres postures fonctionnent de façon plus souterraine et tiennent aux dispositions que les personnes portent avec elles et qu'elles finissent par incarner (conjugaisons de topos liés aux nombreux groupes d'appartenance des personnes et qui cristallisent certaines dispositions notamment sociologiques et de genre).

Cette position n'est d'ailleurs pas incompatible avec celle défendue par Chevallard (1992) lorsqu'il introduit la notion de rapport-personnel et institutionnelaux objets: «Une personne $\mathrm{x}$ est assujettie à une foule d'institutions. Je poserai ici l'axiome qu'une personne n'est en fait rien d'autre que l'émergent d'un complexe d'assujettissements institutionnels. Ce que l'on nomme «liberté» de la personne apparaît alors comme l'effet obtenu en jouant un ou plusieurs assujettissements institutionnels contre d'autres» (p. 91). Ce que cette analyse évacue, ce sont les systèmes d'organisation et de capitalisation des expériences que se donne le sujet/ agent pour devenir une personne.

Le contrat didactique, représente le travail de la transposition interne à la classe, par opposition aux maillons externes de la chaîne transpositive. En suivant le programme didactique et l'étude ascendante de la transposition didactique, nous considérons que le contrat didactique est un système d'attentes spécifiques à une organisation de connaissances. Ce concept est nécessaire pour comprendre: l'action et le fonctionnement du sujet/agent (professeur-P et élève-E) en situation d'enseignement/apprentissage; les caractérsitiques évolutives des systèmes de tâches (milieu potentiel au sens de Brousseau) pour enseigner et apprendre.

Le modèle de l'action conjointe enseignant-élève (Sensevy, Mercier \& Schubauer-Leoni 2000, 2002, 2005; Mercier, Sensevy \& Schubauer-Leoni, 2000; Schubauer-Leoni, Ligozat, Leutenegger, Mercier \& Sensevy, 2004; Sensevy, Schubauer-Leoni, Mercier, Ligozat \& Perrot, 2005 ; Sensevy, Ligozat, Leutenegger \& Mercier, à paraittre) considère trois axes analytiques:

A) L'axe relatif à la construction de la référence: il s'agit ici de décrire l'évolution des objets du milieu, objets qui, en tant que système référentiel en train de se construire, soutiennent les actions conjointes P-E, mais aussi que, par retour, ces actions contribuent à définir au fil de l'activité. Cette co-construction permet aux contributeurs de se situer par rapport à quelque chose qui fait texte et qui est destiné à être consulté. Sa compatibilité avec les pratiques qui font référence en dehors de l'interaction didactique est un enjeu non négligeable. Cet axe est appelé mésogénétique.

B) L'axe relatif aux temporalités (axe dit chronogénétique): on cherche ici à définir la fonction des objets pour enseigner (temps didactique) versus pour apprendre (temps de l'apprentissage) dans le déroulement de l'activité. Les négociations visent, dans ce cas, à : 
- accélérer/ralentir le temps didactique à propos de l'organisation de l'activité - désigner/identifier les objets sensibles du contrat didactique à un moment $t$.

C) L'axe relatif aux positionnements respectifs du professeur et des élèves et leur fonction dans la gestion personnelle (privée/publique) de l'organisation de l'activité (axe dit topogénétique).

Au fur et à mesure de l'avancement des travaux (pour l'essentiel encore dans le domaine des mathématiques), nous avons été amenées à considérer que l'axe mésogénétique commande aux deux autres axes (topo- et chronogénétiques). Ceci a comme conséquence la priorité attribuée, dans l'ordre des analyses, aux descriptions de l'évolution des objets qui viennent à exister dans la co-construction par les agents. Par ailleurs, le modèle de l'action conjointe interroge le fonctionnement du contrat didactique à plusieurs points de vue: celui des représentations, des traces sémiotisées du système d'objets; celui du statut (notamment en termes de recevabilité) des manifestations de connaissance; celui de la diffusion/confrontation au sein du collectif des formes opératoires et prédicatives des connaissances; celui de l'institutionnalisation de certaines connaissances exprimées par les élèves; enfin, celui de conjuguer le double jeu organisationnel de l'activité du professeur et de celles des élèves.

L'ensemble de ce jeu contractuel est censé comporter: la modification des connaissances personnelles; la transformation des sujets à travers diverses expériences d'agentivité; la construction des personnes (processus d'émancipation, autonomisation, émergence d'une posture d'acteur); la viabilité et le renouvellement des institutions. L'analyse a priori de l'activité mathématique (au plan des tâches prescrites) est dans ce cas réinterrogée à la lumière de l'analyse de l'activité mathématique effective des co-agents.

\section{Ouverture en guise de conclusion}

Sans pouvoir développer plus longuement la présentation de ce champ de travaux sur l'action conjointe de l'enseignant et des élèves, nous insistons encore sur les éléments d'articulation entre cette approche de la transposition et celle privilégiée dans le programme épistémologique. Dans les deux cas une importance certaine est attribuée aux contenus culturels censés être diffusés à travers l'institution scolaire. Mais le primat porté aux organisations mathématiques et à son impact sur les organisations didactiques tend à oblitérer les sujets et leurs systèmes d'organisation des rapports évolutifs aux objets. Le mouvement ascendant du programme didactique à visée comparatiste pourrait souffrir, quant à lui, d'une perte de vue de l'organisation des objets de savoir et se trouver ainsi démuni pour saisir l'espace culturel et institutionnel dont disposent effectivement les sujets pour agir. 
Par ailleurs, si nous estimons utile de quitter l'univers des mathématiques enseignées pour prendre en compte à la fois les réalités des pratiques d'enseignement et d'apprentissage des autres disciplines ainsi que les cadrages théoriques que leurs didactiques se sont donnés nous constatons que cette démarche, fort utile pour éviter les effets de réification que produit l'absence de décentration, complexifie grandement le jeu d'articulation entre un programme épistémologique (étude a priori de l'emboîtement de contraintes qui caractérisent l'activité spécifique à un domaine de savoir) et un programme didactique (action conjointe du professeur et des élèves à propos des différents contenus d'enseignement).

Le débat inter-didactiques a encore du grain à moudre pour qu'il soit possible de décanter les composantes génériques du processus de transposition didactique et la part du spécifique. L'identification de telles composantes suppose d'ailleurs que l'on distingue les catégories de spécificité/généricité dont on parle. En effet, une centration sur les objets et leur organisation épistémologique et didactique peut amener à identifier des composantes des pratiques d'enseignement et d'apprentissage qui traversent ou transcendent les contenus tout en étant partiellement affectées par eux; mais on peut aussi suivre le fil des agents, voire des personnes concrètes, en fonction des caractéristiques groupales par ailleurs (le capital culturel hérité, le genre, la place -pour les élèves- dans l'échelle d'excellence de la classe selon les disciplines, les idéologies et théories professionnelles des enseignants,...). L'étude de l'action conjointe de l'enseignant et des élèves est nécessairement appelée à traiter de ces questions si elle veut poursuivre son exploration au-delà du territoire des quelques objets mathématiques qu'elle a mis, pour l'instant, sur le métier de la recherche.

L'enjeu est certes théorique et méthodologique, mais il est indispensable aussi pour que le chercheur didacticien puisse contribuer, sur le terrain, à poser des bases plus solides pour penser le travail co-disciplinaire à l'école.

\section{Notes}

1 Dans l'ouvrage de 85, Chevallard (p. 40) se réfere à Verret (1975, pp. 140-144) pour ce qui est de " la notion de transposition didactique en général " tout en construisant sa propre théorie transpositive dans le domaine des mathématiques.

2 L'entretien mené avec Verret par Terrisse et Léziart (1997) est significatif à ce propos.

3 Vernant (1997) distingue les actions collectives («qui résultent d'un commun accord sur un objectif, des buts, une stratégie») des actions communes («répondant à une intentionnalité collective manifestée par le choix d'un même objectif et d'une même stratégie») et des actions conjointes. Ces dernières, guidées par une intention, conjointe également, font généralement appel à un accord "rapidement fixé ou lentement négocié sur un objectif commun». Si une analyse des œuvres peut nous amener à penser en termes d'action collective, dans le cas de la transposition didactique, dès lors qu'il y a intention (voire mandat officiel à la noosphère) de penser ce qui devra faire l'objet d'enseignement, l'action oblige l'ajustement du projet selon un accord négocié.

4 La théorie de la transposition didactique intégrée au programme de l'approche anthropologique du didactique a trouvé un développement fertile en Espagne grâce aux travaux de Marianna Bosch et Josep Gascon en didactique des mathématiques. 
5 Nous ne pointons pas ici un enjeu libertaire (au sens philosophique) mais plutôt une posture épistémologique sur laquelle nous reviendrons plus loin. Chevallard lui-même introduit une marge de jeu dans le positionnement des sujets lorsqu'il convoque la notion de «rapport personnel» aux objets dans les développements ultérieurs de la théorie de la transposition didactique.

6 Johsua (1998) appelle «savants» les savoirs qui disposent, à un moment historique donné, d'une communauté, généralement académique, habilitée à «dire le vrai» sur les savoirs en question; et considère, par contraste, des savoirs «experts» ceux qui se développent dans d'autres institutions sans qu'ils ne disposent (encore) d'une légitimité suffisamment reconnue pour prétendre au monopole de la véridicité à propos des productions de savoir concernant tel domaine. Il cite à cet égard la conduite des hauts fourneaux, le «résumé de texte» mais aussi la didactique comme théorie sur l'enseignement!

7 Martinand (1987) a introduit à ce propos la notion de pratique sociale de référence en convoquant sous ce label des activités de production, d'ingénierie, domestiques "comparable " à celles mises en œuvre dans les contextes scolaires.

8 Vision récusée explicitement par Chevallard lui-même, notamment dans un texte de 1997: "[...] le mot savoir [...] a pu créer un malentendu malheureux, en confortant la métaphore de la connaissance comme substance[...]. Contre une vision substantialiste des savoirs, je souligne donc que c'est par métonymie, en désignant le tout par une de ses parties, qu'on dira, par exemple, que les mathématiques sont un savoir [...]. Les mathématiques, en effet, sont d'abord une pratique sociale autour de certains objets, pratique dont l'enjeu spécifique est de produire des connaissances, c'est-à-dire des réponses à certaines questions que l'on se pose ..." (p. 25) ]

9 Le double phénomène de décontextualisation-recontextualisation rend compte de la dynamique descendante de la transposition: le savoir (effectivement) enseigné a fait l'objet d'une extraction de son contexte d'origine (lieu d'invention, de production) pour venir prendre place (re-contextualisation) dans une nouvel univers (formé par l'organisation des savoirs à enseigner) apte à rendre cet objet «étudiable» par les élèves, qui se donneront, si possible, les moyens de l'apprendre (à leur tour les élèves vont re-contextualiser le savoir dans le système de tâches constitutif de leur travail d'étudiant et ce faisant ils réaménagent l'organisation déjà-là de leurs connaissances personnelles). Le phénomène de dépersonnalisation-repersonnalisation est intimement lié au précédent. En effet, le travail de dé-recontextualisation engendre le détachement de l'objet des instances (incarnées dans des individus concrets) qui l'ont porté, inventé, travaillé indépendamment et avant qu'il ne soit apprêté pour l'étude de l'élève. Celui-ci lorsqu'il prend à sa charge les questions relatives à ce savoir repersonnalise l'enjeu de connaissance. Ce double processus de contextualisation et de personnalisation affecterait inévitablement aussi le travail de l'enseignant lorsqu'il prend la responsabilité d'extraire des manuels et autres documents didactiques les tâches, les problèmes, les questions jugés aptes à soutenir son action enseignante et partant l'action apprenante des élèves. Guy Brousseau (1998) s'exprime dans ce sens dans son exposé du triple travail du mathématicien, de l'élève et du professeur (pp. 48-50).

10 Une connaissance dite "privée» ne renvoie pas à une vision solipsiste, elle a pour caractéristique d'être au service des sujets qui la manient «à leur niveau» sans pouvoir vérifier la reconnaissance de sa pertinence au plan collectif. Pour les sujets en position d'apprenant, elle est surtout "privée», momentanément, des indices de compatibilité avec le savoir attendu par les sujets en position d'enseignants.

11 Les études d'ingénierie didactique ont été pensées dans cette optique épistémologique.

12 L'expression est de Bosch et Chevallard (1999).

13 Ces organisations sont décrites selon quatre niveaux emboîtés: les types de tâches, les techniques pour la réalisation de ces tâches et, à des niveaux plus «méta», les éléments technologiques et théoriques qui interviennent pour décrire et justifier les pratiques concernant ces tâches. 
14 Bosch et Chevallard (1999) rappellent (p. 115) que l'approche cognitive de Duval porte sur «le fonctionnement de la connaissance sous l'angle des mécanismes et des processus qui la permettent en tant qu'activité d'un être individuel» (Duval, 1996, p. 375).

15 Les propos inhérents à l'action prennent racine dans les travaux que nous conduisons dans l'équipe de didactique comparée à Genève ainsi que dans les multiples échanges avec Alain Mercier et Gérard Sensevy au sein de la communauté naissante des didacticiens comparatistes, et, plus particulièrement, dans le projet, initié par Gérard Sensevy, d'élaboration d'un modèle de l'action du professeur. Ces développements théoriques trouvent par ailleurs des sources d'approfondissement dans les travaux conduits par Jean-Paul Bronckart et collaborateurs visant une théorisation de vaste portée sur les conditions d'émergence de l'agir en lien avec les discours (Bronckart, Bulea, Fillittasz, Fristalon, Plazaola Giger \& Revaz, 2004).

16 Nous distinguons ici les expériences produites sous le contrôle d'ingénieries de celles dites ordinaires. Les situations scolaires ordinaires, c'est-à-dire celles qu'il est possible de rencontrer dans les classes qui ne sont pas en train d'expérimenter une séquence dans le cadre d'un projet d'ingénierie, comportent des professeurs qui mettent au point, eux aussi, de multiples occasions pour favoriser la production d'expériences visant à modifier les connaissances des élèves. Or, divers travaux révèlent le problème institutionnel (et non pas personnel) relatif aux «manques à savoir» (Mercier, 1997; Ligozat 2003) dans le domaine des connaissances mathématiques ainsi que par rapport au comment les élèves apprennent les mathématiques. La science didactique devra se résoudre à chercher une articulation entre les deux catégories de phénomènes suivants: d'un côté (et il s'agit de l'objet privilégié de la didactique des disciplines actuelle), comment se fait-il, alors que les séquences et le situations ont été épistémologiquement contrôlées et calibrées, qu'il y ait toujours autant d'élèves déclarés en difficulté et qui semblent donc "ne pas comprendre» (attribution professorale); d'autre part, comment se fait-il que, dans des circonstances épistémologiquement pauvres voire peu fondées du point de vue mathématique, il y ait, d'après les attributions professorales, des élèves qui apprennent! Voilà que le programme didactique ascendant rejoint les préoccupations du programme épistémologique descendant.

17 Dans le modèle n'existent que des potentiels co-agents. Même lorsque l'élève est appelé à réaliser la tâche en solitaire ("Vous travaillez individuellement», disent les enseignants), les composantes de la situation socialisent la posture de l'agent, le situent dans un projet collectif nécessitant des gestes coopératifs (visant le but commun): pensons à la co-présence des camarades de classes censés réaliser le même travail, l'usage d'instruments culturellement partagés, la nécessité ou la possibilité de remettre le travail accompli à un agent (pair ou enseignant) qui en vérifiera la pertinence.

\section{Références bibliographiques}

Amade-Escot, Ch. (Ed.). (2003). Didactique de l'éducation physique. Etat des recherches. Paris: Editions Revue EPS.

Astolfi, M. (2005). Problèmes scientifiques et pratiques de formation. In O. Maulini \& C. Montandon (Ed.), Les formes de l'éducation: variété et variations (Raisons Educatives pp. 65-81) Bruxelles: De Boeck Université.

Audigier, F. (Ed.). (1988). Actes du colloque Savoirs enseignés et savoirs savants. Paris: INRP.

Audigier, F., Crémieux, C. \& Tutiaux-Guillon, N. (1994). La place des savoirs scientifiques dans les didactiques de l'histoire et de la géographie. Revue Française de Pédagogie, 106, 11 23.

Bautier, E. \& Rochex, J.-Y. (2004). Activité conjointe ne signifie pas significations partagées. In C. Moro \& R. Rickenmann (Ed.), Situation éducative et significations (Raisons Educatives pp. 199-220). Bruxelles: De Boeck Université. 
Beitone, A. \& Legardez, A. (1995). Enseigner les sciences économiques: pour une approche didactique. Revue Française de Pédagogie, 112, 33-45.

Beitone, A., Decugis, M.-A., Dollo, C. \& Rodrigues, C. (2004). Les sciences économiques et sociales. Enseignement et apprentissage. Bruxelles: De Boeck.

Bosch, M. \& Chevallard, Y. (1999). La sensibilité de l'activité mathématique aux ostensifs. Objet d'étude et problématique. Recherches en Didactique des Mathématiques, 19 (1), 77 123.

Bosch, M. \& Gascon, J. (2002). Organiser l'étude. 2. Théorie \& empirie. In. J.-L. Dorier, M. Artaud, M. Artigue, R. Berthelot \& R. Floris (Ed.), Actes de la 1 lème Ecole d'Eté de didactique des mathématiques- Corps 21-30 août 2001 (pp. 23-40). Grenoble: La Pensée Sauvage.

Bosch, M., Espinoza, L. \& Gascon, J. (2003). El profesor como director de procesos de estudio: analisis de organ izaciones didacticas espontaneas. Recherches en Didactique des $\mathrm{Ma}$ thématiques, 23 (1), 79-136.

Bosch, M., Fonseca, C. \& Gascon, J. (2004). Inconpletitud de las organizaciones matematicas locales en las instituciones escolares. Recherches en Didactique des Mathématiques, 24 (2-3), 205-250.

Bronckart, J.-P. (1997). Activité langagière, textes et discours. Pour un interactionnisme socio-discursif. Lausanne, Paris: Delachaux et Niestlé.

Bronckart, J.-P (2000). La psychologie ne peut être que sociale et la didactique est l'une de ses disciplines majeures. In J.-P. Bernié (Ed.), Mélanges offerts à Michel Brossard (pp. 18-41). Bordeaux: Presses Universitaires de Bordeaux.

Bronckart, J.-P., Bulea, E., Filliettaz, L., Fristalon, I., Plazaola Giger, I. \& Revaz, F. (2004). Agir et discours en situation de travail. Cahiers de la Section des sciences de l'éducation, Université de Genève, FPSE, 103.

Bronckart, J.-P. \& Plazaola Giger, I. (1998). La transposition didactique - histoire et perspectives d'une problématique fondatrice. Pratiques, 97/98, 35-38.

Bronckart, J.-P. \& Schneuwly, B. (1991). La didactique du Français langue maternelle: l'émergence d'une utopie indispensable. Education et recherche, 13 (1), 8-26.

Brousseau, G. (1998). Théorie des situations didactiques, en mathématiques. Grenoble: La Pensée Sauvage.

Bruner, J. (1991). Car la culture donne forme à l'esprit. Paris: ESHEL

Canelas Trevisi, S. (1997). La transposition didactique dans les documents pédagogiques et dans les interactions en classe. Thèse de doctorat, Université de Genève, FPSE.

Canelas-Trevisi, S., Moro, Ch., Schneuwly, B. \& Thévenaz. Th. (1999). L’objet enseigné: vers une méthodologie plurielle d'analyse des pratiques d'enseignement en classe. Repères 20, 143-162.

Charaudeau, P. (2004). Comment le langage se noue à l'action dans un modèle socio-communicationnel du discours. De l'action au pouvoir. In L. Filliettaz (Ed.), Les modèles $d u$ discours face au concept d'action. Actes du 9ème Colloque de Pragmatique de Genève et Colloque Charles Bally (Cahiers de linguistique française 26, pp. 151-175). Genève: Département de Linguistique, Faculté des Lettres, Université de Genève.

Chatel, E. (Ed.). (1995). Transformation des savoirs en sciences économiques et sociales. Revue française de pédagogie, 112, 9-20.

Chevallard, Y. (1985/1991). La transposition didactique. Du savoir savant au savoir enseigné. Grenoble: La Pensée Sauvage.

Chevallard, Y. (1989). Le concept de rapport au savoir, rapport personnel, rapport institutionnel, rapport officiel. Actes du séminaire de didactique (pp. 211-235). Grenoble: IMAG.

Chevallard, Y. (1992). Concepts fondamentaux de la didactique: perspectives apportées par une approche anthropologique. Recherches en didactique des mathématiques, 12 (1), 73-112.

Chevallard, Y. (1997). L'enseignement des SES est-il une anomalie didactique? Skholé, 6, 2537. 
Dollo, C. (2005). Généricité/spécificité d'un concept: la transposition didactique en sciences économiques et sociales. Revue suisse des sciences de l'éducation, 27 (1), 85-102.

Duval, R. (1996). Quel cognitif retenir en didactiques des mathématiques? Recherches en didactiques des mathématiques, 16 (3), 349-382.

Friedrich, J. (1999). La rencontre Leontiev-Vygotski: quelques concepts clés. In Y. Clot (Ed.), Avec Vygotski (pp. 141-163). Paris: La Dispute.

Gascon, J. (1998). Evolución de la didáctica de las matemáticas como disciplina cientifica. Recherches en didactiques des mathématiques, 18 (1), 7-34.

Halté, J.-F. (1992). La didactique du français. Paris: PUF Coll. Que sais-je?

Halté, J.-F. (1998). L'espace didactique et la transposition. Pratiques, 97, 171-192.

Johsua, S. (1985). Contribution à la délimitation du contraint et du possible dans l'enseignement de la physique. Essai de didactique expérimentale. Thèse d'Etat, Université d'Aix-Marseille 2.

Johsua, S. (1996). Le concept de transposition n'est-il propre qu'aux mathématiques? In C. Raisky \& M. Caillot (Ed.), Au delà des didactiques, le didactique. Débats autour de concepts fédérateurs (pp. 61-73). Paris, Bruxelles: De Boeck.

Johsua, S. (1998). Des "savoirs» et de leur étude: vers un cadre de réflexion pour l'approche didactique. L'année de la recherche en sciences de l'éducation 1999 (pp. 79-97). Paris: PUF.

Leutenegger, F. (2000). Construction d'une «clinique» pour le didactique. Une étude des phénomènes temporels de l'enseignement. Recherches en didactique des mathématiques, 20 (2), 209-250.

Leutenegger, F. (2003). Etude des interactions didactiques en classe de mathématiques: un prototype méthodologique. In A. Danis, M.L. Schubauer-Leoni \& A. Weil-Barais (Ed.), Interaction, acquisition de connaissances et développement (Bulletin de psychologie, tome 56(4), 466, pp. 559-571). Paris: Bulletin de psychologie.

Ligozat, F. (2003). Apprendre à écrire des "grands nombres»: la place des pratiques langagières dans une leçon de mathématiques. In M. Jaubert, M. Rebière \& J.-P. Bernié (Ed.), Construction des connaissances et langage dans les disciplines d'enseignement. Colloque pluridisciplinaire, Bordeaux 3-5 avril 2003.[CD-ROM]

Marschall, M., Plazaola Giger, I., Rosat, M.-C. \& Bronckart, J.-P. (2000). La transposition didactique des notions énonciatives dans les manuels d'enseignement des langues vivantes. Fribourg: Editions universitaires.

Martinand, J.-L. (1987). Pratiques de référence, transposition didactique et savoirs professionnels en sciences et techniques. Les sciences de l'éducation pour l'ère nouvelle, 1-2, 23-30.

Mercier, A. (1997). La relation didactique et ses effets. In C. Blanchard-Laville (Ed.), Variations sur une leçon de mathématiques. Analyses d'une séquence: "L'écriture des grands nombres" (pp. 259-312). Paris: L'Harmattan.

Mercier, A. (2002). La transposition des objets d'enseignement et la définition de l'espace didactique, en mathématiques. Note de synthèse. Revue Française de pédagogie, 141, 135-171.

Mercier, A., Schubauer-Leoni, M-L, \& Sensevy, G. (Ed.). (2002). Vers une didactique comparée. Revue Française de pédagogie 141, 5-16.

Mercier, A., Sensevy, G., \& Schubauer-Leoni, M.-L. (2000). How Social Interactions within a Class Depend on the Teacher's Assessment of the Various Pupils' Mathematical Capabilities, a Case Study. Zentblatt für Didaktik der Mathematik, International Review of Mathematics Education, 6(32), 126-130.

Raisky, C., Caillot, M. (Ed.). (1996). Au delà des didactiques, le didactique. Débats autour de concepts fédérateurs. Paris, Bruxelles: De Boeck.

Rogalski, J. (2004). La didactique professionnelle: une alternative aux approches de « cognition située " et " cognitiviste " en psychologie des acquisitions, @ctivités, 1 (2), 103-120 [Page Web]. Accès: (2), 103-120. http://www.activites.org/v1n2/Rogalski.pdf

Rogalski, J. \& Samurcay, R. (1994). Modélisation d'un «savoir de référence» et transposition didactique dans la formation de professionnels de haut niveau. In G. Arsac, Y. Chevallard, 
J.-L. Martinand \& A. Tiberghien (Ed.), La transposition didactique à l'épreuve (pp. 35-71). Grenoble: La Pensée Sauvage.

Schneuwly, B. (1994). Genres et types de discours: considérations psychogénétiques et ontogénétiques. In Y. Reuter (Ed.), Les interactions lecture-écriture (pp. 155-173). Berne: Peter Lang.

Schneuwly, B. (1995). De l'utilité de la «transposition didactique». In J.-L. Chiss, J. David \& Y. Reuter (Ed.), Didactique du français, état d'une discipline (pp. 47-62). Paris: Nathan.

Schubauer-Leoni, M.-L. (1998). L'évaluation didactique: une affaire contractuelle. In J. Weiss (Ed.), L'évaluation: problème de communication (pp. 79-95). Fribourg: Del Val.

Schubauer-Leoni, M.-L. \& Leutenegger, F. (2002). Expliquer et comprendre dans une approche clinique/expérimentale du didactique «ordinaire». In F. Leutenegger \& M. SaadaRobert (Ed.), Expliquer et comprendre en Sciences de l'Education (Raisons Educatives, pp. 227-251). Paris, Bruxelles: De Boeck Université.

Schubauer-Leoni, M.-L, Leutenegger, F. \& Mercier, A. (1999). Interactions didactiques dans l'apprentissage des "grands nombres». In M. Gilly, J.-P. Roux \& A. Trognon (Ed.), Apprendre dans l'interaction. Analyse des médiations sémiotiques (pp. 301-328). Nancy et Aixen-Provence: Presses Universitaires de Nancy \& Publications de l'Université de Provence.

Schubauer-Leoni, M.-L., Ligozat, F., Leutenegger, F., Mercier, A \& Sensevy, G. (2004). Capire l'azione dell'insegnante per interpretare l'attività dell'allievo in classe. In B. D'Amore et S. Sbaragli (Ed) La didattica della matematica una scienza per la scuola (pp.49-64). Bologna: Pitagora editrice.

Sensevy, G. (2001). Théories de l'action et action du professeur. In J.-M. Baudouin \& J. Friedrich (Ed.), Théories de l'action et éducation (Raisons Educatives, pp. 203-224). Paris, Bruxelles: De Boeck Université.

Sensevy, G., Ligozat, F., Leutenegger, F. \& Mercier, A. (2005) The teacher's action, the researcher's conception in mathematics. In CERME 4 Proceedings "Teaching practices to issues in teacher's education" (workgroup 12), Feb, 17th-21st. 2005, Sant Feliu de Guixols, Spain. [Page Web]. Accès: http://cerme4.crm.es/

Sensevy, G., Mercier, A. \& Schubauer-Leoni, M.-L. (2000). Vers un modèle de l'action didactique du professeur à propos de la course à 20. Recherches en didactique des mathématiques 20 (3), 263-304.

Sensevy, G., Mercier, A. \& Schubauer-Leoni, M-L. (2002). A Model for Examining Teachers' Didactic Action in Mathematics, The Case of the Game "Race to 20", Proceedings of European Research in Mathematics Education II, Marienbad, Février 2001.

Sensevy, G., Schubauer-Leoni, M-L., Mercier, A., Ligozat, F. \& Perrot, G., (2005). An Attempt to Model the Teacher's Action in Math Class. Educational Studies in mathematics, 59, 153-181.

Tall, D. (Ed.). (1991). Advanced mathematical Thinking. Dordrecht: Kluwer.

Terrisse, A. \& Léziart, Y. (1997). L'émergence d'une notion: la transposition didactique. Entretiens avec Michel Verret. Les sciences de l'éducation pour l'ère nouvelle, 3, 5-27.

Tiberghien, A. (1989). Transposition didactique, le cas de la physique. In G. Arsac, M. Develay \& A. Tiberghien (Ed.), La transposition didactique en mathématiques, en physique et en biologie (pp. 37-57). IREM de Lyon et LIRDIS, Université Claude Bernard.

Tozzi, M. (1995). De la philosophie à son enseignement: le sens d'une didactisation. In M. Develay (Ed.), Savoirs scolaires et didactiques des disciplines, une encyclopédie pour aujourd'hui (pp. 237-260) Paris: ESF.

Veillard, L. (2000). Le rôle des situations professionnelles dans la formation en alternance, cas des élèves-ingénieurs de production de l'ISTP de Saint-Etienne. Thèse de doctorat en Sciences de l'Education, Université Lumière Lyon II.

Vernant, D. (1997). Du discours à l'action. Paris: PUF

Verret, M. (1975). Le temps des études. Thèse d'état, Université de Paris V. Paris: Librairie Honoré Champion. 
Mots clés: Transposition didactique, action du professeur, contrat didactique, mésogenèse, chronogenèse, topogenèse

\section{Ein zweiter Blick auf die Besonderheiten der didaktischen Übermittlung im Licht der vergleichenden Didaktik}

\section{Zusammenfassung}

Die Phänomene der didaktischen Übermittlung nehmen einen wichtigen Platz in der Untersuchung des Lehr-Lern-Prozesse ein. Diese Theorie von Y. Chevallard in den 1980er Jahren wollten die anderen Didaktiken nach kritischer Prüfung in ihre eigenen Fachbereiche integrieren. Ausgehend von der mathematischen Didaktik hat die Theorie sich, ohne ihr epistemologisches Programm zu vernachlässigen, in der praktischenAusführung in Richtung eines anthropologischen Ansatzes entwickelt. Dieser Artikel ist aufgrund einer Umfrage der vergleichenden Didaktik entstanden und hat zum Ziel, sowohl die wichtigsten Aspekte als auch die Schwachpunkte zu beleuchten. Ein besonderes Augenmerk wird gerichtet auf die Frage der Funktionen der «Personalisation» und der «Kontextualisation» im Hinblick auf Untersuchungen der Didaktik im Schulalltag.

Schlagworte: didaktische Umsetzung; Lehrerhandeln, didaktischer Vertrat, Mesogenesis, Chronogenesis; Topogenesis

\section{Una rilettura dei fenomeni traspositivi alla luce della didattica comparata}

\section{Riassunto}

I fenomeni inerenti alla trasposizione didattica sono centrali nei processi d'insegnamento e di apprendimento. Questa teoria, iniziata da Y. Chevallard negli anni ' 80 , è stata discussa dalle altre didattiche disciplinari che hanno tentato di importarla nel loro campo. A partire dalla didattica della matematica, la teoria si è sviluppata verso un approccio antropologico delle pratiche didattiche inoltrandosi più avanti nel suo programma epistemologico. Questo articolo, nato da un insieme di domande sorte nel campo della didattica comparata, cerca di mettere in luce certi aspetti fondamentali della teoria ma anche dei punti oscuri o impensati. Si discute in modo particolare la questione del ruolo attribuito ai fenomeni detti di «personalizzazione» e di «contestualizzazione» nella prospettiva di studiare ciò che di regola succede nelle classi dal punto di vista didattico.

Parole chiave: Trasposizione didattica, azione dell'insegnante, contratto didattico, mesogenesi, cronogenesi, topogenesi 


\section{Re-considering transposition phenomena from a comparative didactics point of view Summary .}

\section{Summary}

In this paper, didactical transposition phenomena are regarded as a main issue in the study of teaching and learning processes. The Didactical Transposition Theory was developed in the 1980's by Y. Chevallard, in order to explain how school mathematics may differ from the body of knowledge arising from the mathematicians' community. Meanwhile, other didactics have attempted to adapt this theory to their own subject matter. Within the didactics of mathematics field, Chevallard's theory was broadened to an anthropologic approach of the didactic practices according to its epistemological rationale. Today, comparative didactics attempts to question both fundamental features and some remaining blind spots in this theory. More particularly, this paper focuses on the "personalization" and "contextualization" phenomena for the purpose of studying the didactical interactions in ordinary classes.

Key words: Didactical transposition phenomena, teachers's action, didactic contract, mesogenesis, chronogenesis, topogenesis 
T h e m a 